ISSN electrónico: 2445-1355

DOI: https://dx.doi.org/10.14201/fj2020525162

\title{
DESARROLLO, ELABORACIÓN Y CONTROL DE UN PREPARADO COSMÉTICO ANTIESTRÍAS
}

\section{Development, Elaboration and Control of an Anti Stretch Cosmetic Preparation}

\section{Rocío GONZÁLEZ PANIAGUA; Aránzazu ZARZUELO CASTAÑEDA}

Facultad de Farmacia. Departamento de Ciencias Farmacéuticas. Área de Farmacia y Tecnología Farmacéutica. Universidad de Salamanca. Campus Miguel de Unamuno. Calle Lic. Méndez Nieto, s/n. 37007 Salamanca

Correo-e: rociogonpan@gmail.com

RESUMEN: Las estrías son cicatrices atróficas formadas por la rotura y pérdida de fibras de colágeno, elastina y fibrina. Actualmente, se han convertido en un problema estético más que médico, que cursan con un gran componente emocional, y que pueden ocasionar síntomas físicos como picazón, sensibilidad y dolor. Se pueden desarrollar a cualquier edad, pero son más frecuentes en el inicio de la pubertad; son más frecuentes en las mujeres y se desarrollan en zonas de crecimiento corporal. Las estrías están condicionadas por múltiples factores endocrinos y exocrinos.

A día de hoy, no existe un tratamiento que elimine por completo a la estría, por lo que se emplean tratamientos orientados a la prevención y atenuacion de la misma. El objetivo de esta memoria es el desarrollo de productos cosméticos con una composición eficaz, combinables con otros tratamientos y de fácil elaboración desde la oficina de farmacia.

Palabras clave: estría; diagnóstico; tratamiento; asiaticósidos; rosa mosqueta; ácido retinoico.

ABSTRACT: Stretch marks are atrophic scars formed by the breakage and loss of collagen, elastin and fibrin fibers. Currently, they have become 
an aesthetic problem rather than medical, wich have a great emotional component and can cause physical symptoms suchs as itching, tenderness and pain. They can develop at any age, but they are more frequent at the onset of puberty; they are more frequent in women and develop in areas of body growth. Stretch marks are conditioned by multiple endocrine and exocrine factors.

Today, there is no treatment that completely eliminates stretch marks, so treatments aimed at preventing and attenuating it are used. The objective of this memory is the development of cosmetic products with an effective composition, combinable with other treatments and easily prepared from the pharmacy office.

Keywords: stetch marks; diagnosis; treatment;asiaticosides; rosehip; retinoic acid.

\section{INTRODUCCIÓN}

Las estrías son cicatrices formadas por el desgarro o distensión de la retícula cutánea o de la membrana extracelular, localizada en la dermis. Esta membrana es muy delicada por lo que se daña con facilidad cuando la piel sufre algún trauma, como roces, distensiones, retrocesos excesivos, tensión o restricción con demasiada velocidad para adecuarse a las dimensiones corpóreas (Cárdenas Vallejo, Rojas Gómez, 2007; Vargas Martínez et al., 2016).

Las estrías, con los canones de belleza actuales, se han convertido en un problema estético más que en un problema médico; aunque no son perjudiciales para la salud, pueden ocasionar síntomas físicos como picazón, sensibilidad y dolor (Adam, Ardeshir, 2017; Ayala, 2000; Hannah, 2018). Algunos expertos recomiendan no trivializar este tipo de condiciones dermatológicas, ya que para algunas personas las estrías pueden tener un impacto psicológico o emocional duradero.

Las estrías se desarrollan en cualquier persona y edad, siendo más frecuentes en un rango de edad entre 12 y 50 años. Son un tercio más frecuentes en la adolescencia, a partir de la cual, si no se tratan, la presencia de las estrías será permanente (Ayala, 2000; Hannah, 2018).

La población femenina es la más afectada y en la que ocasionan una mayor preocupación e impacto emocional. Las distintas situaciones hormonales que sufre la mujer, como la pubertad, el embarazo, fármacos hormonales, dietas, etc., hacen que la aparición de las estrías sea mayor en las mujeres que en los hombres (Ayala, 2000; Azcana, 2009; Hannah, 2018). 
Generalmente, su aparición se relaciona con grandes y distintas zonas corporales como la región periaxilar, mamaria, periumbilical, fosas iliacas, lumbar, muslos y región poplítea (Pardo Vicuña, 2017; S. Ud, McGeorge, 2016; Vargas Martínez et al., 2016). Igualmente, es muy frecuente que esta atrofia cutánea se presente en la piel junto con otras anomalías como celulitis, varices, flacidez, etc., lo que empeora el estado de ánimo de las personas que las sufren.

Histológicamente, las estrías son una forma de cicatrización atrófica que afecta especialmente a fibras de colágeno, elastina y fibrina. Se producen por la rotura y pérdida de fibras de colágeno, ocasionando una disminución de cohesión cutánea. Como consecuencia, se cede el área a las fuerzas de tensión de masas musculares que soporta la piel (Arylide, 2019).

El origen de las estrías está relacionado con factores tanto endocrinos como exocrinos y otros factores: neurofisiológicos (representados por el sistema hipotálamo-hipófisis suprarrenal y el sistema simpático-medular suprarrenal), mecánicos, estrés, etc.; por tanto, la tensión cutánea no es la causa principal de la aparición de las estrías (Arylide, 2019; Ayala, 2000; Azcana, 2009; Cárdenas Vallejo, Rojas Gómez, 2007; Vargas Martínez et al., 2016).

Los factores endocrinos están conformados por 2 hormonas, las corticosuprarrenales y los estrógenos (Ayala, 2000; Cárdenas Vallejo, Rojas Gómez, 2007; Pardo Vicuña, 2017; Vargas Martínez et al., 2016).

Los factores mecánicos dan lugar a una distensión más duradera del tejido debido a un aumento del tejido subyacente, el cual es diferente en función de las distintas situaciones de la persona (Ayala, 2000; Cárdenas Vallejo, Rojas Gómez, 2007; Vargas Martínez et al., 2016):

- Tejido adiposo y conjuntivo, aumento en embarazo, obesidad y celulitis.

- Tejido muscular, aumento en atletas.

Otros factores que desencadenan la aparición de estrías son el estrés, el Síndrome de Ehlers-Danlos, la herencia, infecciones que producen liberación de toxinas, estados de inmunodeficiencia, enfermedades (tifus, tuberculosis), anorexia nerviosa, obesidad, insuficiencia hepática, etc. (Ayala, 2000; Cárdenas Vallejo, Rojas Gómez, 2007; Pardo Vicuña, 2017; Vargas Martínez et al., 2016).

El proceso evolutivo de las estrías comprende 2 fases, caracterizadas por (Adam, Ardeshir, 2017; Cárdenas Vallejo, Rojas Gómez, 2007; Misticone, Piquero, 2007; Vargas Martínez et al., 2016):

- Fase aguda o antiinflamatoria, fase inicial, en la que las estrías se manifiestan como lesiones rosáceas (estría rubra) y elevadas. Las estrías pueden evolucionar a la siguiente fase.

- Fase cicatricial o crónica, en la que las estrías se presentan como depresiones a atrofias dérmicas hipopigmentadas (estría alba). 
Actualmente, el tratamiento eficaz para la eliminación de las estrías ya formadas no existe, sino que se trata de un tratamiento dedicado a la profilaxis, fundamentalmente en la fase inflamatoria, y a la atenuación de la estría en la fase crónica (Ayala, 2000; Azcana, 2009; Cárdenas Vallejo, Rojas Gómez, 2007; Hannah, 2018). El objetivo de la prevención es el mantenimiento y mejora dérmica, lo que consigue una dermis elástica, con capacidad para resistir el estiramiento cutáneo (Pardo Vicuña, 2017). El tratamiento es diferente en función de la fase en la que se encuentre la estría (Adam, Ardeshir, 2017; Ayala, 2000; Azcana, 2009; Cárdenas Vallejo, Rojas Gómez, 2007; elfarmacéutico, 2011; Mayo clinic, 2019; Pardo Vicuña, 2017):

- Fase inflamatoria: consiste en un tratamiento farmacológico y cosmético, basado en una solución de indometacina con estimulantes de glicosaminoglicanos. Los productos antiestrías más utilizados son hidrolizados de colágeno y elastina; asiaticósidos; ácido hialurónico; vitaminas A, B, C, $\mathrm{D}$, E; extractos placentarios, entre otros.

- Fase cicatricial: consiste en un tratamiento combinado, basado en retinoides tópicos; ácido glicólico; Tretinoína; Láser IR; Láser He-Ne acompañado de extractos de canela, algas, cinc...; peelings, entre otros.

Los resultados del tratamiento dependen de múltiples factores, como son las características de las estrías, la antigüedad de las mismas, la composición del cosmético, su forma de aplicación, etc.

En cuanto a composición, no solo es importante una correcta elección de los ingredientes cosméticos, sino también la función del excipiente que debe retrasar la absorción, ya que, si es muy rápida, los efectos beneficiosos no se conseguirán de forma completa (Ayala, 2000; elfarmacéutico, 2011).

Si después de un tratamiento dermocosmético no se consigue un resultado favorable, se pueden utilizar otros tratamientos más invasivos, como corticoides intralesionales, cirugía, láser, etc. (elfarmacéutico, 2011).

\section{2. Овjetivo}

El objetivo de este trabajo ha sido el diseño y elaboración de un producto cosmético que aporte una composición eficaz para el tratamiento en ambas fases de las estrías y combinable, si es preciso, con tratamientos farmacológicos. Dicha formulación debe ser fácilmente abordable desde la oficina de farmacia, aportando nuevos productos al tratamiento cosmético existente y permitiendo su personalización en función de los requerimientos personales. 


\section{Materiales y métodos}

- Estudio bibliográfico. Para llevar a cabo este trabajo, inicialmente se realizó una revisión bibliográfica para conocer las características de la patología a tratar y los ingredientes cosméticos más adecuados para llevar a cabo la formulación de un cosmético adaptado a dichas necesidades. Dicha búsqueda se realizó en los buscadores Google académico y Pubmed. Así mismo, se obtuvo información a través de revistas científicas como Elsevier y a través de páginas oficiales de profesionales sanitarios.

La búsqueda se acotó a los últimos 10 años y principalmente a los 4 últimos.

- Estudio de estabilidad. Las formulaciones elaboradas, adecuadamente acondicionadas en envases airless, se almacenan en cámaras a $5{ }^{\circ} \mathrm{C}$, $25^{\circ} \mathrm{C} / 60 \% \mathrm{HR}$ y $30{ }^{\circ} \mathrm{C} / 65 \%$ HR durante 6 meses, realizándose los siguientes controles:

o Variación de peso. Especificación: $\pm 5 \%$.

o pH: según procedimiento PN/L/CP/001/00. Formulario Nacional 2. ${ }^{\mathrm{a}}$ ed. [Madrid]: Ministerio de la Presidencia; 2015.

o Evanescencia: según procedimiento PN/L/CP/003/00.

o Microbiología: según Real Farmacopea Española.

\section{Resultados}

Previo al diseño de la formulación, se realizó una búsqueda de ingredientes cosméticos con funcionalidad antiestría, quedando recogidos en la Tabla 1 aquellos que se consideraron más interesantes (Adam, Ardeshir, 2017; Ayala, 2000; Cárdenas Vallejo, Rojas Gómez, 2007; Misticone, Piquero, 2007; Vargas Martínez et al., 2016).

Una vez seleccionados los ingredientes cosméticos, se diseñó la primera fórmula (emulsión $\mathrm{O} / \mathrm{W}$ ), seleccionando excipientes hipoalergénicos y no irritantes (Fichas técnicas de Acofarma; Ficha técnica de Hidroxiprolisilano).

La fórmula elaborada presentaba un olor muy fuerte debido al árbol del té, difícil de enmascarar, por lo que se decidió eliminar dicho ingrediente, planteándonos la inclusión del Hidroxipropilsilano CN debido a sus 3 acciones principales: estimula la biosíntesis de colágeno, actúa de forma acentuada sobre los procesos regenerativos y normaliza la renovación celular (Ficha técnica de Hidroxiprolisilano), pero, debido a su no disponibilidad a través de los principales laboratorios suministradores de ingredientes cosméticos, se descartó su utilización. En su lugar, se incorporaron ácido glicólico y ácido retinoico, ya que estos 2 compuestos de forma conjunta han demostrado un efecto beneficioso en la patología en estudio. Igualmente se incluyó vitamina E por su acción antioxidante, regenerante, protectora e hidratante a nivel dermoepidérmico. 
TABLA 1. Ingredientes cosméticos más interesantes con funcionalidad antiestría

\begin{tabular}{|c|c|}
\hline $\begin{array}{l}\text { Aceite de rosa mosqueta } \\
\text { (Rosa rubiginosa, Rosa } \\
\text { eglanteria) }\end{array}$ & $\begin{array}{l}\text {-Alto contenido en ácidos grasos esenciales } \\
\text { (linoleico y linolénico): regeneración de tejidos. } \\
\text {-Actividad hidratante, aceleración de cicatrización, } \\
\text { estimulación de angiogénesis, antiinflamatoria, } \\
\text { prevención y atenuación de estrías, eliminación de } \\
\text { manchas, etc. }\end{array}$ \\
\hline \multirow{3}{*}{$\begin{array}{l}\text { Extracto de centella asiática } \\
\text { (Centella asiática }[\mathrm{L}] \\
\text { Urban) }\end{array}$} & $\begin{array}{l}\text {-Elementos plásticos, aminoácidos (alanina y } \\
\text { prolina): síntesis endógena de elastina y colágeno. }\end{array}$ \\
\hline & $\begin{array}{l}\text {-Actividad hidratante, reparadora, reestructurante, } \\
\text { bioestimulante, antiinflamatoria, antivaricosa, } \\
\text { anticelulítica y aceleradora de la cicatrización. }\end{array}$ \\
\hline & $\begin{array}{l}\text { - Mecanismo de acción: estimulación de } \\
\text { proliferación de fibroblastos y producción de fibras } \\
\text { de colágeno y elastina. }\end{array}$ \\
\hline \multirow{4}{*}{$\begin{array}{l}\text { Ácido glicólico (derivado de } \\
\text { la caña de azúcar) }\end{array}$} & -Potenciador acción humectantes \\
\hline & $\begin{array}{l}\text { Protección del colágeno y elastina, producción } \\
\text { de peeling no abrasivo, eliminación de manchas, } \\
\text { incremento de hidratación cutánea, estimulación de } \\
\text { circulación sanguínea, etc. }\end{array}$ \\
\hline & $\begin{array}{l}\text { - Mejora el estado de estrías: mecanismo } \\
\text { desconocido. }\end{array}$ \\
\hline & $\begin{array}{l}\text { - Mejora la matriz dérmica: aumento de síntesis de } \\
\text { ácido hialurónico y sustancias intercelulares, con } \\
\text { aumento del grosor dérmico. }\end{array}$ \\
\hline \multirow{3}{*}{$\begin{array}{l}\text { Elastina (proteína en tejido } \\
\text { conectivo, sistema muscular, } \\
\text { huesos y articulaciones) }\end{array}$} & $\begin{array}{l}\text { Constituida por aminoácidos (prolina y valina) y } \\
\text { minerales (glicina y magnesio). }\end{array}$ \\
\hline & $\begin{array}{l}\text {-Actividades de hidratación, elasticidad y } \\
\text { resistencia, que promueven flexibilidad y retención } \\
\text { de agua. }\end{array}$ \\
\hline & $\begin{array}{l}\text {-Aportación de numerosos nutrientes: contribución } \\
\text { a la elasticidad, fuerza y firmeza. }\end{array}$ \\
\hline \multirow{3}{*}{$\begin{array}{l}\text { Vitamina E o tocoferol } \\
\text { (vitamina liposoluble) }\end{array}$} & $\begin{array}{l}\text {-Actividad antioxidante: prevención de oxidación de } \\
\text { constituyentes celulares esenciales. }\end{array}$ \\
\hline & $\begin{array}{l}\text {-Actividad antiinflamatoria importante: regenerar a } \\
\text { nivel dermoepidérmico. }\end{array}$ \\
\hline & $\begin{array}{l}\text { Contribución en mantenimiento de hidratación de } \\
\text { piel y en retraso de envejecimiento general cutáneo. }\end{array}$ \\
\hline
\end{tabular}




\begin{tabular}{|l|l|}
\hline & $\begin{array}{l}\text { Producción de mayor grosor de dermis y } \\
\text { epidermis: activación del colágeno y fibroblastos. } \\
\text {-Regulación de diferenciación celular con } \\
\text { incremento en fibroplasia, colagenosis y } \\
\text { angiogénesis. }\end{array}$ \\
$\begin{array}{l}\text { Ácido retinoico (derivado } \\
\text { de la vitamina A } \\
\text { liposoluble) }\end{array}$ & $\begin{array}{l}\text {-Receptores de ácido hialurónico influidos por } \\
\text { ácido retinoico: mayor elasticidad y rehidratación } \\
\text { cutánea. } \\
\text {-Presencia de proteína ligadora celular para el ácido } \\
\text { retinoico en epidermis y fibroblastos y receptores } \\
\text { en genes específicos. } \\
\text {-Actividad de peeling químico. }\end{array}$ \\
\hline
\end{tabular}

Tras elaborar la segunda fórmula, el resultado fue una emulsión de consistencia muy líquida, por lo que se modificó la proporción de los componentes grasos (lanolina, ácido esteárico y alcohol cetílico). Como consecuencia del aumento de la proporción de lanolina, el aspecto de la emulsión era graso y poco cosmético, por lo que se prescindió de dicho excipiente en las posteriores composiciones, variando la proporción de ácido esteárico y de alcohol cetílico para ajustar la composición final dentro de una proporción de fase grasa/acuosa de 25/75.

Otro cambio con respecto a la composición inicial fue el cambio de nipasol por phenoxip XB debido al mayor espectro de actividad, mayor estabilidad, baja toxicidad y por no afectar al color y olor de las fórmulas.

Tras probar distintos porcentajes, se eligió la emulsión con proporción $5 \%$ de ácido esteárico y $5 \%$ de alcohol cetílico, debido a su consistencia ideal para el objetivo de estudio.

En una segunda fase se diseñó una formulación en textura cremigel, debido a que esta textura permite una mayor extensibilidad y evanescencia, además de poder refrescante y descongestivo. Entre las preferencias de un producto cosmético antiestrías se incluye su facilidad de extensión, una mayor fluidez y no dejar restos sobre piel y ropa.

Como gelificante se utilizó sepigel, el cual está compuesto por una mezcla de polímero acrílico, isoparafina y emulgente, que permite obtener geles con una concentración muy baja, con afinidad con componentes grasos y de forma instantánea con una buena consistencia y sin adhesividad. Se elaboraron 2 cremigeles con distintas proporciones de sepigel ( $3 \%$ o $5 \%$ ), con el objetivo de obtener la textura deseada, siendo la proporción del $5 \%$ la que ofrecía la consistencia buscada.

Las distintas fórmulas y sus composiciones quedan recogidas en la Tabla 2. 
Una vez decididas las fórmulas se elaboraron lotes de mayor tamaño, los cuales se envasaron en envase airless y se almacenaron en cámaras climáticas a las condiciones comentadas anteriormente, con el fin de conocer la estabilidad de la fórmula.

Las formulaciones diseñadas, para poder ser consideradas cosmético, tienen que tener un $\mathrm{pH}$ superior a 4,2-4,4 por la presencia de ácido glicólico; un $\mathrm{pH}$ inferior (que aumenta la actividad de los a-hidroxiácidos) hace que la formulación sea considerada medicamento. Las formulaciones propuestas se elaboraron a los dos $\mathrm{pH}$ con el fin de conocer su estabilidad y ampliar la fórmula de cosmético a medicamento.

Tabla 2. Composición de la emulsión $\mathrm{O} / \mathrm{W}$ antiestrías y del cremigel

\begin{tabular}{|c|c|c|c|c|c|c|}
\hline Ingrediente & Función & $\begin{array}{l}\text { Fórmula } 1 \\
\text { emulsión } \\
\text { O/W (\%) }\end{array}$ & $\begin{array}{c}\text { Fórmula } 2 \\
\text { emulsión } \\
\text { O/W } \\
\%)\end{array}$ & $\begin{array}{c}\text { Fórmula } 3 \\
\text { emulsión } \\
\text { O/W( } \\
\%)\end{array}$ & $\begin{array}{l}\text { Fórmula } 1 \\
\text { cremigel ( } \\
\%)\end{array}$ & $\begin{array}{c}\text { Fórmula } 2 \\
\text { cremigel } \\
(\%)\end{array}$ \\
\hline Agua & Solvente & $\begin{array}{c}\operatorname{csp} \\
100,00\end{array}$ & $\operatorname{csp} 100,0$ & $\operatorname{csp} 100,0$ & $\operatorname{csp} 100,0$ & $\operatorname{csp} 100,0$ \\
\hline $\begin{array}{l}\text { Aceite del } \\
\text { árbol del té }\end{array}$ & Cicatrizante & 2,0 & & & & \\
\hline $\begin{array}{l}\text { Aceite } \\
\text { de rosa } \\
\text { mosqueta }\end{array}$ & $\begin{array}{l}\text { Regenerador, } \\
\text { atenuación } \\
\text { de cicatrices, } \\
\text { estimulante }\end{array}$ & 8,0 & 8,0 & 8,0 & 8,0 & 8,0 \\
\hline $\begin{array}{l}\text { Ácido } \\
\text { cítrico }\end{array}$ & $\begin{array}{l}\text { Potencia } \\
\text { antioxidante, } \\
\text { regulador de } \mathrm{pH}, \\
\text { tamponante }\end{array}$ & 1,0 & 1,0 & 1,0 & & \\
\hline $\begin{array}{l}\text { Ácido } \\
\text { esteárico }\end{array}$ & $\begin{array}{c}\text { Emoliente, } \\
\text { emulgente, } \\
\text { protector, no } \\
\text { desecación de capa } \\
\text { córnea }\end{array}$ & 10,0 & 4,0 & 5,0 & & \\
\hline $\begin{array}{l}\text { Ácido } \\
\text { glicólico }\end{array}$ & $\begin{array}{l}\text { Antienvejecimiento, } \\
\text { estimulante de } \\
\text { renovación celular }\end{array}$ & & 3,0 & 5,0 & 5,0 & 5,0 \\
\hline $\begin{array}{l}\text { Ácido } \\
\text { retinoico }\end{array}$ & $\begin{array}{l}\text { Hidrorrestitutiva, } \\
\text { regeneración } \\
\text { de colágeno, } \\
\text { prevención }\end{array}$ & & 0,1 & 0,1 & 0,1 & 0,1 \\
\hline
\end{tabular}


ROCÍO GONZÁLEZ PANIAGUA; ARÁNZAZU ZARZUELO CASTAÑEDA

DESARROLLO, ELABORACIÓN Y CONTROL DE UN PREPARADO COSMÉTICO ANTIESTRÍAS

\begin{tabular}{|c|c|c|c|c|c|c|}
\hline Ingrediente & Función & $\begin{array}{l}\text { Fórmula } 1 \\
\text { emulsión } \\
\text { O/W (\%) }\end{array}$ & $\begin{array}{c}\text { Fórmula } 2 \\
\text { emulsión } \\
\text { O/W ( } \\
\%)\end{array}$ & $\begin{array}{c}\text { Fórmula } 3 \\
\text { emulsión } \\
\text { O/W ( } \\
\%)\end{array}$ & $\begin{array}{l}\text { Fórmula } 1 \\
\text { cremigel ( } \\
\%)\end{array}$ & $\begin{array}{c}\text { Fórmula } 2 \\
\text { cremigel } \\
(\%)\end{array}$ \\
\hline $\begin{array}{l}\text { Alcohol } \\
\text { cetílico }\end{array}$ & $\begin{array}{c}\text { Emulgente, } \\
\text { emoliente, } \\
\text { opacificante, } \\
\text { absorbente de agua, } \\
\text { mejora consistencia }\end{array}$ & 10,0 & 3,0 & 5,0 & 5,0 & 5,0 \\
\hline Elastina & $\begin{array}{c}\text { Mejora consistencia } \\
\text { y elasticidad }\end{array}$ & 5,0 & 5,0 & 5,0 & 5,0 & 5,0 \\
\hline $\begin{array}{c}\text { Extracto } \\
\text { de centella } \\
\text { asiática }\end{array}$ & $\begin{array}{l}\text { Cicatrizante, } \\
\text { estimulante }\end{array}$ & 5,0 & 5,0 & 5,0 & 5,0 & 5,0 \\
\hline Glicerina & $\begin{array}{l}\text { Preservante, } \\
\text { antimicrobiana, } \\
\text { emoliente, } \\
\text { humectante, } \\
\text { disolvente }\end{array}$ & 15,0 & 15,0 & 14,0 & 10,0 & 10,0 \\
\hline Lanolina & $\begin{array}{l}\text { Hidratante, } \\
\text { emoliente }\end{array}$ & 5,0 & 2,0 & & & \\
\hline $\begin{array}{l}\text { Miristato de } \\
\text { isopropilo }\end{array}$ & $\begin{array}{c}\text { Vehículo, } \\
\text { lubricante de piel y } \\
\text { acondicionamiento, } \\
\text { aglutinante, } \\
\text { emoliente, } \\
\text { disolvente } \\
\end{array}$ & 2,0 & 2,0 & 6,0 & & \\
\hline Nipasol & Conservante & 0,3 & 0,3 & & & \\
\hline $\begin{array}{l}\text { Phenoxip } \\
\text { XB }\end{array}$ & Conservante & & & 0,3 & 0,4 & 0,4 \\
\hline Sepigel & Gelificante & & & & 5,0 o 3,0 & 5,0 \\
\hline Tween 80 & $\begin{array}{c}\text { Protector, emoliente, } \\
\text { retención de agua, } \\
\text { emulsionante no } \\
\text { iónico }\end{array}$ & 10,0 & 10,0 & 6,0 & & \\
\hline Vitamina E & $\begin{array}{c}\text { Regenerador, } \\
\text { protector, } \\
\text { hidratante a nivel } \\
\text { dermoepidérmico, } \\
\text { antioxidante }\end{array}$ & & 0,5 & 0,5 & 0,5 & 0,5 \\
\hline
\end{tabular}

Los resultados de los ensayos realizados sobre ambas formulaciones ponen de manifiesto que ambas formulaciones son estables en las condiciones de estudio durante seis meses, quedando recogidos los resultados obtenidos en la Tabla 3. 
TABla 3. Resultados de los ensayos realizados sobre la emulsión O/W antiestrías y cremigel antiestrías

\begin{tabular}{|c|c|c|c|c|}
\hline & Parámetro & Tiempo cero & $\begin{array}{l}\text { Tiempo seis } \\
\text { meses }\end{array}$ & \\
\hline & & & $5^{\circ} \mathrm{C}$ & $25^{\circ} \mathrm{C} / 60 \% \mathrm{HR}$ \\
\hline \multirow{5}{*}{$\begin{array}{l}\mathrm{E} \\
\mathrm{M} \\
\mathrm{U} \\
\mathrm{L} \\
\mathrm{S} \\
\mathrm{I} \\
\text { Ó } \\
\mathrm{N}\end{array}$} & $\begin{array}{c}\text { Aspectos y características } \\
\text { organolépticas }\end{array}$ & \multicolumn{3}{|c|}{$\begin{array}{l}\text { Crema fluida, de tono amarillento, olor poco } \\
\text { perceptible y agradable. }\end{array}$} \\
\hline & $\begin{array}{c}\mathrm{pH} \\
\text { Cosmético: } \\
\text { Medicamento: }\end{array}$ & $\begin{array}{l}4,6 \\
2,9\end{array}$ & $\begin{array}{l}4,7 \\
2,9\end{array}$ & $\begin{array}{l}4,8 \\
2,8\end{array}$ \\
\hline & Homogeneidad & \multicolumn{3}{|c|}{$\begin{array}{l}\text { Homogénea, sin presencia de grumos, gotículas de } \\
\text { agua ni burbujas de aire. }\end{array}$} \\
\hline & Evanescencia & \multicolumn{3}{|c|}{ Evanescente y de fácil aplicación } \\
\hline & $\begin{array}{l}\text { Variación de peso ( \%) } \\
\text { Cosmético: } \\
\text { Medicamento: }\end{array}$ & - & $\begin{array}{l}0,8 \\
1,2\end{array}$ & $\begin{array}{l}0,7 \\
0,6\end{array}$ \\
\hline $\begin{array}{l}\mathrm{O} \\
/ \\
\mathrm{W}\end{array}$ & Microbiología & \multicolumn{3}{|c|}{$\begin{array}{l}\text { Aerobios totales: } \leq 10^{2} \mathrm{CFU} / \mathrm{ml} \text {. } \\
\text { Hongos: } \leq 10 \mathrm{CFU} / \mathrm{ml} \text {. } \\
\text { Pseudomonas aeruginosa: ausencia/g. } \\
\text { Staphylococcus aureus: ausencia/g. }\end{array}$} \\
\hline \multirow{6}{*}{$\begin{array}{c}\mathrm{C} \\
\mathrm{R} \\
\mathrm{R} \\
\mathrm{M} \\
\mathrm{I} \\
\mathrm{G} \\
\mathrm{E} \\
\mathrm{L}\end{array}$} & $\begin{array}{c}\text { Aspecto y características } \\
\text { organolépticas }\end{array}$ & \multicolumn{3}{|c|}{$\begin{array}{l}\text { Crema fluida, de tono amarillo, olor poco perceptible } \\
\text { y agradable. }\end{array}$} \\
\hline & $\begin{array}{c}\mathrm{pH} \\
\text { Cosmético: } \\
\text { Medicamento: }\end{array}$ & $\begin{array}{l}4,8 \\
2,8\end{array}$ & $\begin{array}{l}4,7 \\
2,8\end{array}$ & $\begin{array}{l}4,8 \\
2,9\end{array}$ \\
\hline & Homogeneidad & \multicolumn{3}{|c|}{$\begin{array}{l}\text { Homogénea, sin presencia de grumos, gotículas de } \\
\text { agua ni burbujas de aire. }\end{array}$} \\
\hline & Evanescencia & \multicolumn{3}{|c|}{ Evanescente y de fácil de aplicación. } \\
\hline & $\begin{array}{l}\text { Variación de peso ( \%) } \\
\text { Cosmético: } \\
\text { Medicamento: }\end{array}$ & - & $\begin{array}{l}1,3 \\
1,1\end{array}$ & $\begin{array}{l}1,1 \\
0,4\end{array}$ \\
\hline & Microbiología & \multicolumn{3}{|c|}{$\begin{array}{c}\text { Aerobios totales: } \leq 10^{2} \mathrm{CFU} / \mathrm{ml} \text {. } \\
\text { Hongos: } \leq 10 \mathrm{CFU} / \mathrm{ml} \text {. } \\
\text { Pseudomonas aeruginosa: ausencia/g. } \\
\text { Staphylococcus aureus: ausencia/g. }\end{array}$} \\
\hline
\end{tabular}

\section{Conclusiones}

La rotura de las fibras de colágeno y elastina da lugar a la aparición de estrías. Durante el proceso de formación de la estría hay distintos tratamientos, aunque actualmente ninguno consigue la eliminación completa de la misma. Sin embargo, cuanto antes se inicie el tratamiento, la respuesta al mismo será más favorable. 
Por ello, los productos desarrollados se presentan como un tratamiento preventivo y como atenuación de la apariencia de la estría, ya sea como medicamentos o como cosméticos. Al tener una composición de ingredientes cosméticos con acción sobre la fase inflamatoria y cicatricial hace que los productos sean aún más eficaces en el tratamiento de las estrías. Además, no se incluyen perfumes ni alcohol y los conservantes son hipoalergénicos.

Las dos formulaciones antiestrías desarrolladas, emulsión $\mathrm{O} / \mathrm{W}$ y cremigel, son productos asequibles, cosméticamente agradables y de fácil elaboración en la oficina de farmacia. Las características cosméticas que presentan son de preferencia, ya que se absorbe rápidamente, no deja residuo graso, de consistencia fluida, no irritante, olor poco perceptible, etc.

Con respecto a la estabilidad se ha puesto de manifiesto que son estables durante 6 meses, de modo que pueden dispensarse con total seguridad.

Existe una gran ventaja frente a los cosméticos presentes en el mercado, basada en la personalización del producto, adaptándolo a los requerimientos del cliente, por ejemplo, según el tipo de piel o de otras patologías/alteraciones que presente.

Es importante recalcar que en el momento de la dispensación hay que hacer especial hincapié en que debe ser aplicado con un masaje, haciendo círculos con las manos para aumentar la circulación local y el aporte de elementos hídricos y plásticos (Ayala, 2000; elfarmacéutico, 2011), con el fin de garantizar que el producto penetre a mayor profundidad y acceda a la zona lesionada. Para empezar a ver resultados favorables, es aconsejable aplicarse el producto cosmético 2 veces al día y de forma continua.

Asimismo, hay que insistir al usuario que debe acompañar el tratamiento tópico de una serie de medidas higiénico-dietéticas, como llevar una dieta saludable (aporte de aminoácidos, vitaminas y minerales como vegetales verdes, germen de trigo, semillas, fruta, soja, etc.) y beber abundante agua, realizar ejercicio, mantener la piel hidratada, evitar la exposición solar, etc. (elfarmacéutico, 2011; Hannah, 2018).

\section{Bibliografía}

Arylide: Pathology of stretch marks [Internet]. Canadá: Arylide; [citado mayo 2019]. Disponible en: http://arylide.com/stratamark/pathology-of-stretch-marks

Ayala A. Las estrías. Etiología, clínica y tratamiento. Offarm. 2000; 19(2):1-152.

Azcana L. Cuidado de la piel en el embarazo. MSPharma. 2009; 23(4):46-50.

Cárdenas Vallejo L, Rojas Gómez LM. Elaboración de crema antiestrías a partir de productos naturales a escala de laboratorio. Medellín; 2007.

elfarmacéutico.com: Estrías y cicatrices [Internet]. España: Ediciones Mayo; 2011 [actualizado marzo 2019; citado abril 2019]. Disponible en: http://elfarmaceutico.es/index. php/la-revista/item/718-estrias-y-cicatrices\#.XIQA8S1DmqQ 
Fichas técnicas de Acofarma. Disponible en: https://www.acofarma.com

Ficha técnica de Hidroxiprolisilano CN. Disponible en: http://www.biosiltech.com/ pdf/exsymol/Hydroxyprolisilane \%20CN \%20- \%20A3 \%20brochure.pdf

Hague A, Bayat A. Therapeutic targets in the management of striae distensae: A systematic review. JAAD. 2017; 77(3):559-568.

Mayo clinic: Stretch marks [Internet]. Inglaterra: Mayo Clinic; 2019. [actualizado noviembre 2018; citado abril 2019]. Disponible en: https://www.mayoclinic. org/diseases-conditions/stretch-marks/diagnosis-treatment/drc-20351144

Misticone S, Piquero V. «Peeling» de ácido retinóico al 7 \% en estrías cutáneas: una opción terapéutica útil y segura. Svderma. 2007; 45(1):18-22.

Nichols H. How do I get rid of stretch marks?. MNT. 2018.

Pardo Vicuña M de L. Evaluación in vivo de la eficacia cosmética de dos procedimientos de bioestimulación con la aplicación de plasma rico en plaquetas sobre estrías, para mejorar la elasticidad y firmeza de la piel tratada. Ecuador; 2017.

Ud-Din S, McGeorge D, Bayat A. Topical management of striae distensae (strech marks): prevention and therapy of striae rubrae and albae. JEADV. 2016; 30(2):211-222.

Vargas Martínez YA et al. Diseñar y formular una crema antiestrías a base de aceites esenciales elaborada en el laboratorio de tecnología de medicamentos de la Facultad de Ciencias Químicas. Nicaragua; 2016. 